\title{
Design of Coal Mine Belt Motor Speed Monitoring System Based on DSP
}

\author{
Yukun Wang ${ }^{1,2, *}$ \\ ${ }^{1}$ Coal Science Group Shenyang Research Institute Co., Ltd., Fushun, Liaoning, 113122, China \\ ${ }^{2}$ State Key Laboratory of Coal Mine Safety Technology, Fushun, Liaoning, 113122, China
}

Corresponding author Email:651779986@qq.com

Keywords: Coal mine, DSP, Belt motor, Optoelectronic encoder, Speed detection.

\begin{abstract}
In order to improve the speed detection accuracy of coal mine belt motor, this paper introduces the principles of digital signal processor (DSP) and photoelectric encoder in detail, and designs the speed monitoring system of coal mine belt motor by using the internal orthogonal coding pulse unit module of DSP, with emphasis on the interface circuit between DSP and photoelectric encoder.
\end{abstract}

\section{Introduction}

In recent years, with the further advancement of resource integration and merger and reorganization of small coal mines in China, the pace of mechanized transformation of coal mines has been accelerated, and the level of coal mine safety production guarantee has been improved, which has greatly promoted the improvement of coal mine safety production situation. However, the progress of coal mine merger, reorganization and integration of technological transformation is uneven among regions, and some coal mines with integrated technological transformation violate regulations or evade closure in the name of integrated technological transformation. There are a lot of hidden dangers in the process of coal mine merger, reorganization, integration and technical transformation. Flammable gases such as gas easily explode when they meet sparks, and accidents in production safety often occur.

Belt conveyor for coal mine has the characteristics of large transportation volume, complex working environment, strong bearing capacity and long transportation distance. It is widely used in Shanxi, Inner Mongolia, Xinjiang and other major coal producing areas in China. Belt conveyor for coal mine can be used not only in coal production and processing, but also in other mineral production and processing. Coal mine belt motor is the power source of coal mine belt conveyor, its safety is directly related to the safety of coal mine conveying system. It is of great significance to carry out the research on the detection technology of coal mine belt motor running speed for improving the reliability of coal mine belt transportation, eliminating potential safety hazards and reducing production accidents.

\section{Design of monitoring system}

Coal mine belt motor monitoring system is mainly composed of data processor system, photoelectric encoder, interface circuit, control panel, display module, communication module, etc. the system structure is shown in Figure 1. Photoelectric encoder is used to collect the speed data of belt motor in coal mine. TMS320F2812 DSP chip is selected for data processor system, which is used to collect feedback signal of photoelectric encoder, calculate and process. The interface circuit is used to convert the output signal of photoelectric encoder into a signal recognizable by DSP. The control panel is used to send control instructions to control the start and stop of the system. The display module is used to display the system working status, speed data and other information. The communication module is used to send the speed data collected by DSP to the superior monitoring system. 


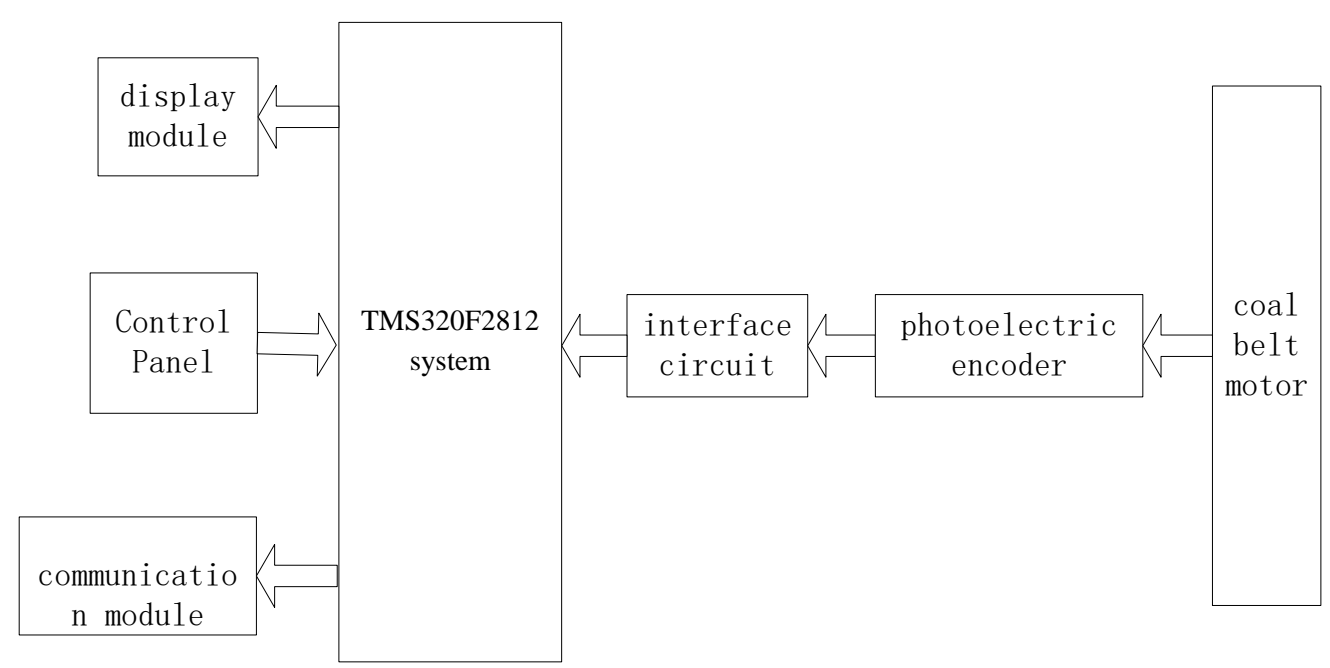

Reality module; Control module; Communication module

TMS320F2812 system

Interface circuit; Photoelectric encoder; Coal mine belt motor

Figure 1. The structure of coal mine belt motor monitoring system

\subsection{TMS320F2812}

TMS320F2812 digital signal processor is the latest 32-bit fixed-point DSP controller and one of the most advanced processors in the control field. The highest processing frequency can reach $150 \mathrm{MHz}$, which improves the response speed and processing accuracy of the control system. TMS320F2812 supports programming in common computer languages such as $\mathrm{C} / \mathrm{C}++$, which is convenient for users to design software. TMS320F2812 has a function library of floating-point data calculation, and realizes floating-point operation on fixed-point processor. TMS320F2812 integrates $128 \mathrm{~KB}$ flash memory, which can effectively prevent the programs in ROM from being stolen by simple programming and 128-bit key during software development and upgrade. Up to 56 independently programmed general-purpose input/output pins, 12-bit 16-channel A/D converter, and single-channel conversion time up to 60ns. On-chip standard communication port can provide simple communication port for host and test equipment, display and other components. TMS320F2812 digital signal processor is widely used in solar power generation, automotive radar and servo motor control.

\subsection{Photoelectric encoder}

Optoelectronic encoder is a sensor that converts the mechanical geometric displacement on the output shaft into pulse or digital quantity by using photoelectric conversion principle. The photoelectric encoder is mainly composed of grating disk and photoelectric detection device, and the grating disk is equally divided into several rectangular holes. In the detection system, the photoelectric disk is coaxial with the detected object, and the detected object rotates at the same speed instead of the photoelectric disk. Several pulse signals are detected by the detection device composed of light-emitting diodes and other electronic components, and the current motor speed can be reflected by calculating the number of pulses output by the photoelectric encoder per second. In addition, the photoelectric encoder provides two channel signals with a phase difference of 90, and the system can judge the turning direction of the motor according to the change of the state of the two channel optical codes. According to the calibration method and signal output form, photoelectric encoders are divided into three types: incremental, absolute and hybrid.

E6B2-CWZ6C incremental rotary encoder is selected as the speed and direction detection device of mine flameproof motor. the resolution of the sensor can reach 1,024ppr, with zero phase, the power supply can reach $24 \mathrm{vdc}$, the output frequency can reach $100 \mathrm{KHz}$, the rotating speed can reach $3000 \mathrm{rpm}$, and the protection level can reach IP54. When the incremental rotary encoder rotates, it 
can continuously output the number of pulses corresponding to the rotation angle, but it does not output at rest, so long as the pulse part is counted, the rotation position can be known. The incremental rotary encoder can choose the reference position, which can be adjusted according to the Z-phase signal which is only output once in one circle. The sensor 1 is shown in fig. 2.

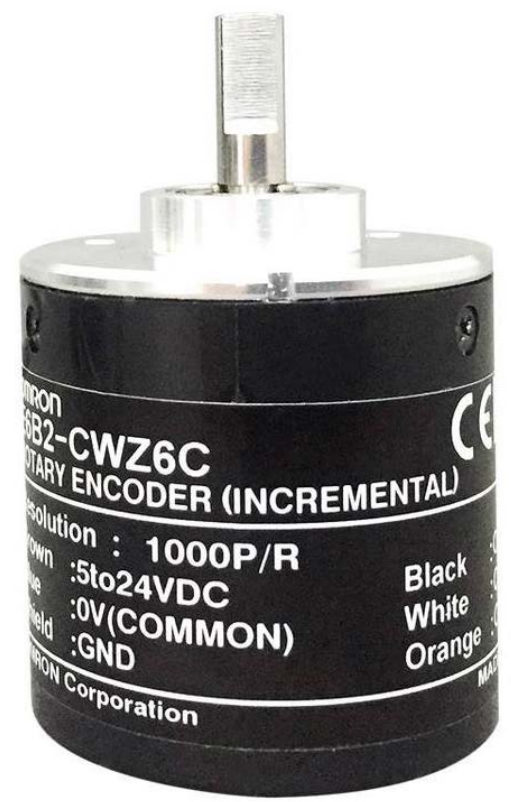

Figure 2. Incremental rotary encoder

\subsection{Interface circuit}

Each event manager module of the DSP chip has a quadrature encoding pulse (QEP) circuit. When the QEP circuit is enabled, the DSP can decode and count the quadrature encoded pulses on the CAP1/QEP1 and CAP2/QEP2 (for EVA) or CAP4/QEP3 and CAP5/QEP4 (for EVB) pins. The QEP circuit can be used to connect a photoelectric encoder to obtain the rotation direction and speed information of the mine flameproof motor. When the QEP circuit is enabled, the capture function of the CAP1/CAP2 and CAP4/CAP5 pins is disabled. The QEP unit is connected to the incremental photoelectric encoder installed on the motor spindle through the signal conversion unit. The DSP decodes and counts the quadrature encoded pulse signal, and realizes the speed and direction of the mine flameproof motor through the interrupt program. Calculation and storage of parameters.

The output signal of photoelectric encoder is $5 \mathrm{~V}$ square wave signal, and the maximum voltage that DSP can receive is $3.3 \mathrm{~V}$, so it is necessary to design a signal conversion circuit to convert $5 \mathrm{~V}$ square wave signal into signal that DSP can receive. The $5 \mathrm{~V}$ square wave signal is isolated by capacitor filter and fast optical coupler 6N137, and then converted into pulse signal which can be received by DSP by dual voltage comparator LM393. The reference voltage of the LM393 comparator is $1.8 \mathrm{~V}$. When the input voltage is greater than the comparator reference voltage, the comparator outputs a high level. The $5 \mathrm{~V}$ regulated power supply is converted by the integrated three-terminal regulator LM317 with adjustable output voltage to provide working power for the comparator. The comparator outputs a pulse signal that can be recognized by the DSP. The signal conversion circuit is shown in Figure 3. 


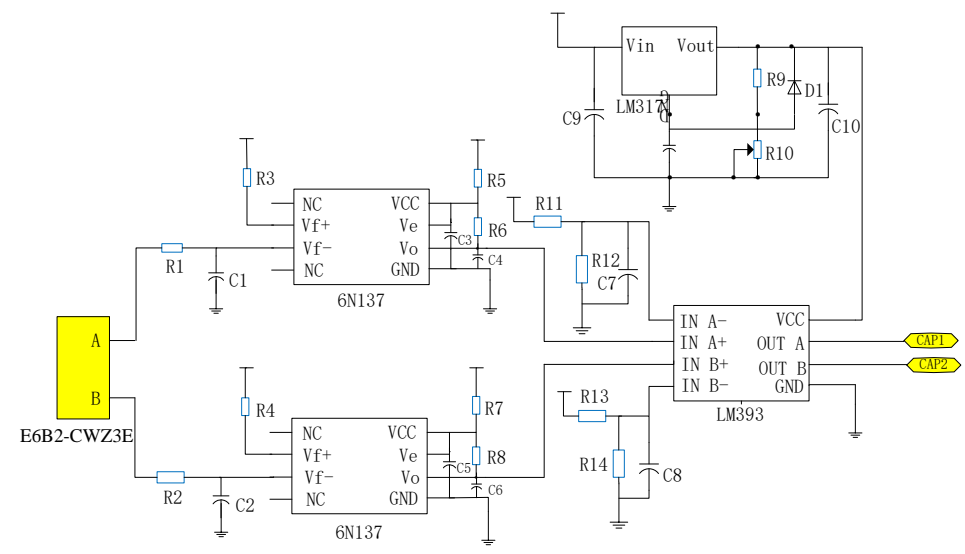

Figure 3. Signal conversion circuit

\section{Conclusions}

Combined with the current situation of coal mine safety production in China, the speed detection system of flameproof motor for mine is designed by combining DSP and photoelectric encoder. The photoelectric encoder is transmitted to the orthogonal coding pulse unit of DSP through the signal conversion circuit, and the direction and speed of the motor can be obtained through internal processing by DSP according to the sequence of double pulses and pulse frequency. The detection system has the advantages of simple structure, high reliability and good real-time performance. It can realize the real-time detection of mine flameproof motor speed and meet the expected design requirements.

\section{References}

[1] DANG Zhen-ping, PEN Zong-ren. Errosion test on 500kV composite rod insulator fractured in service and its electric tress estimation. Insulators and Surge Arresters, No.4,1-7,2005.

[2] Xin Lin, De-shun Wang, Jian-yuan Xu. Linear Servo Motor Operating Mechanism and Control Technique for High-voltage Circuit Breaker. Proceedings of the CSEE, vol.27,pp. 137-141,2008.

[3] GAO Bo, ZHANG Ya-ting, WANG Qing-liang, et al. Effect of pollution non-uniformity on electric field of contaminated insulator. Insulators and Surge Arresters, No3,13-16,2008.

[4] Yongxiang Li, Xin Lin, Jianyuan Xu. Design of a Novel Permanent Magnet Brushless DC Motor-Driven Operating Mechanism for High-Voltage Circuit Breaker and Its Dynamic Simulation. Power System Technology, vol. 1, 185-189,2010.D. Z. Cheng, Controllability of switched bilinear systems, IEEE Trans. on Automatic Control, Vol.50, No.4, 511-515, 2005.

[5] WEN Kang-zhen, WEN Yuan-fang, DUANMU Lin-nan, et al. The error of ANSYS solution of electrostatic field produced by sharp electrode. Insulators and Surge Arresters, No.2,14-16,2009. 